IP/BBSR/95-66

hep-th/9507063

\title{
S-DUALITY AND COSMOLOGICAL CONSTANT IN STRING THEORY
}

\author{
Supriya $\mathrm{KaR}^{1}$, Jnanadeva Maharana ${ }^{2}{ }^{*}$ And Harvendra Singh ${ }^{2} \dagger$ \\ ${ }^{1}$ Mehta Research Institute of Mathematics 85 Mathematical Physics, \\ 10, Kasturba Gandhi Marg, Allahabad-211 002, India \\ ${ }^{2}$ Institute of Physics, Bhubaneswar 751 005, India
}

\begin{abstract}
The S-duality invariance of the equations of motion of four dimensional string effective action with cosmological constant, $\Lambda$, is studied. It is demonstrated that the S-duality symmetry of the field equations are broken for nonzero $\Lambda$. The "naturalness" hypothesis is invoked to argue that $\Lambda$ should remain small since exact S-duality symmetry will force the cosmological constant to vanish in the string effective action.
\end{abstract}

( To appear in Phys. Lett. B )

*Jawaharlal Nehru Fellow

†e-mail: maharana, hsingh@iopb.ernet.in 
It is recognized that S-duality is an important symmetry of string theory which relates the strong and weak coupling domains. The consequences of this symmetry [1] are interesting and surprising. In the recent past, a variety of novel results have been derived for supersymmetric gauge theories [2] in sequel to these new developments in string theory.

The purpose of this article is to explore further the consequences of S-duality. It is well known that the equations of motion derived from the effective action are invariant under S-duality, although the action is not. However, these results are derived in the absence of the cosmological constant term in the action. We show that in presence of the cosmological constant, $\Lambda$, the equations of motion associated with a four dimensional effective action, obtained through dimensional reduction, are not invariant under S-duality transformations. Nevertheless, the invariance of the equations of motion is recovered once we set $\Lambda=0$. This leads us to conjecture that exact S-duality symmetry will force the cosmological constant to vanish. At this stage, we are tempted to invoke the hypothesis of naturalness [3] expounded by 't Hooft which says that a parameter in any theory remains small, if the symmetry is enhanced by setting that parameter to zero. For example, in electrodynamics setting the electron mass $m_{e}=0$ enhances the symmetry of the action and the chiral symmetry is restored. Therefore, it is guaranteed that $m_{e}$ remains small and the corrections are proportional to $m_{e}$ itself. We recall that according to 't Hooft, the vanishingly small value of cosmological constant is unnatural [3]; putting it equal to zero does not seem to increase the symmetry of Einstein-Hilbert action. Consequently, he concluded that gravitational effects do not obey naturalness in conventional theory. However in the framework of string theory, $\Lambda$ obeys the naturalness criterion, i.e. putting $\Lambda=0$ enhances the stringy symmetry. We are aware that S-duality is not a symmetry of the action and hence may not satisfy the strict criterion of naturalness.

The cosmological constant is a parameter measured very close to zero and it is a vintage theoretical problem to explain the smallness of $\Lambda$ which has eluded physicists for a long time [4]. Several attempts have already been made to explain the vanishing cosmological constant in the framework of string theory [5]. Recently, Witten [6] has argued that the vanishing of 
cosmological constant and the absence of massless dilaton might be explained by a duality between supersymmetric string vacuum in three dimensions and a non-supersymmetric string vacuum in four-dimensions. The issue has also been addressed in a more concrete model by Becker, Becker and Strominger [7].

In this investigation, we study four-dimensional string effective action in the presence of cosmological constant term. First, we consider an effective action which is derived by toroidal compactification of a higher dimensional action. The massless field contents of the resulting four dimensional superstring action in the bosonic sector are: graviton, antisymmetric tensor, dilaton, gauge fields and the moduli. In four dimensions, the antisymmetric tensor field can be traded for a pseudoscalar axion $\chi$. The dilaton, axion and gauge fields transform nontrivially under S-duality whereas the metric and moduli remain invariant. The Einstein and matter field equations are derived and it is shown that these equations are not invariant under S-duality transformations. Next, we consider an effective action which admits charged black hole solutions. It is well known that, near the horizon, the underlying conformal field theory is described by tensor product of two WZW models: one corresponds to an $S L(2, R)$ whereas the other is identified with an $S U(2)$ with WZW lavel constants $K_{S L}$ and $K_{S U}$ respectively. The corresponding four dimensional string effective action is recognized as an action obtained from the compactification of a six dimensional one with a cosmological constant term. We present yet another way to obtain the four dimensional black hole solution by axial gauging of the $S L(2, R)$ WZW Lagrangian. The four dimensional effective action is derived and explicit solutions of the background field equations are given.

Now, consider a string effective action in $D$ spacetime dimensions with massless fields such as graviton, $\hat{G}_{M N}$, antisymmetric tensor, $\hat{B}_{M N},(M, N=0,1, \cdots, D-1)$, dilaton, $\hat{\Phi}$ and $n$ Abelian gauge fields, $\hat{A}_{M}^{I}$. If we compactify coordinates on a $d=D-4$ dimensional torus and assume that the backgrounds are independent of these $d$ compact coordinates, the resulting four dimensional reduced effective action takes the following form [8], 


$$
\begin{aligned}
S=\int d^{4} x \sqrt{-g} e^{-\Phi}\left(R+g^{\mu \nu} \partial_{\mu} \Phi \partial_{\nu} \Phi\right. & +\frac{1}{8} \operatorname{Tr}_{\mu} M^{-1} \partial^{\mu} M \\
& \left.-\frac{1}{4} \mathcal{F}_{\mu \nu}^{i}\left(M^{-1}\right)_{i j} \mathcal{F}^{j \mu \nu}-\frac{1}{12} H_{\mu \nu \lambda} H^{\mu \nu \lambda}-2 \Lambda\right),
\end{aligned}
$$

where

$$
\begin{aligned}
& \hat{G}_{M N}=\left(\begin{array}{cc}
g_{\mu \nu}+A_{\mu \alpha}^{(1)} A_{\nu}^{(1) \alpha} & A_{\mu \beta}^{(1)} \\
A_{\nu \alpha}^{(1)} & G_{\alpha \beta}
\end{array}\right), \\
& M=\left(\begin{array}{ccc}
G^{-1} & -G^{-1} C & -G^{-1} A^{T} \\
-C^{T} G^{-1} & G+C^{T} G^{-1} C+A^{T} A & C^{T} G^{-1} A^{T}+A^{T} \\
-A G^{-1} & A G^{-1} C+A & 1+A G^{-1} A^{T}
\end{array}\right),
\end{aligned}
$$

$C_{\alpha \beta}=\frac{1}{2} A_{\alpha}^{I} A_{\beta}^{I}+B_{\alpha \beta}$ and $\Phi=\hat{\Phi}-\frac{1}{2} \ln \operatorname{det} G_{\alpha \beta}$ is shifted dilaton with the spacetime dependent background fields $\left(G_{\alpha \beta}, A_{\alpha}^{I} \equiv \hat{A}_{\alpha}^{I}, B_{\alpha \beta} \equiv \hat{B}_{\alpha \beta}\right)$ defining a generic point in moduli-space in the toroidal compactification of string theory. The moduli $\mathrm{M}$ is a $(2 d+n) \times(2 d+n)$ matrix valued scalar field and satisfies the condition $M \mathcal{L} M \mathcal{L}=1$, where $\mathcal{L}$ is the $O(d, d+n)$ metric,

$$
\mathcal{L}=\left(\begin{array}{ccc}
0 & I_{d} & 0 \\
I_{d} & 0 & 0 \\
0 & 0 & I_{n}
\end{array}\right), \quad \Omega^{T} \mathcal{L} \Omega=\mathcal{L}
$$

Here $I_{d}$ is d-dimensional identity matrix and $\Omega$ is an element of the group $O(d, d+n)$. The field strengths appearing in (1) are

$$
\begin{aligned}
H_{\mu \nu \lambda} & =\partial_{\mu} B_{\nu \lambda}-\frac{1}{2} \mathcal{A}_{\mu}^{i} \mathcal{L}_{i j} \mathcal{F}_{\nu \lambda}^{j}+\text { cyclic perm } \\
\mathcal{F}_{\mu \nu}^{i} & =\partial_{\mu} \mathcal{A}_{\nu}^{i}-\partial_{\nu} \mathcal{A}_{\mu}^{i}
\end{aligned}
$$

where i, j are $O(d, d+n)$ matrix indices. $\mathcal{A}_{\mu}^{i} \equiv\left(A_{\mu}^{(1) \alpha}=\hat{G}_{\mu \alpha}, A_{\mu \alpha}^{(2)}=\hat{B}_{\mu \alpha}+\hat{B}_{\alpha \beta} A_{\mu}^{(1) \beta}+\right.$ $\left.\frac{1}{2} \hat{A}_{\alpha}^{I} A_{\mu}^{(3) I}, A_{\mu}^{(3) I}=\hat{A}_{\mu}^{I}-\hat{A}_{\alpha}^{I} A_{\mu}^{(1) \alpha}\right)$ is a $(2 d+n)$ component vector field. It is more convenient for the implementation of S-duality transformation to rescale the $\sigma$-model metric to Einstein metric, $g_{\mu \nu} \rightarrow e^{\Phi} g_{\mu \nu}$, and introduce the axion $\partial_{\sigma} \chi=\left(\eta^{2} / 6\right) \sqrt{-g} \epsilon_{\mu \nu \lambda \sigma} H^{\mu \nu \lambda}$ where $\eta=e^{-\Phi}$. Then (1) can be reexpressed as

$$
\begin{aligned}
S=\int d^{4} x \sqrt{-g}\left(R-\frac{1}{2 \eta^{2}} g^{\mu \nu} \partial_{\mu} \Psi \partial_{\nu} \bar{\Psi}\right. & +\frac{1}{8} \operatorname{Tr}\left(\partial_{\mu} M^{-1} \partial^{\mu} M\right) \\
& \left.-\frac{1}{4} \eta \mathcal{F}_{\mu \nu}^{i} M_{i j}^{-1} \mathcal{F}^{j \mu \nu}+\frac{1}{4} \chi \mathcal{F}_{\mu \nu}^{i} \mathcal{L}_{i j} \tilde{\mathcal{F}}^{j \mu \nu}-\frac{2 \Lambda}{\eta}\right),
\end{aligned}
$$


where $\Psi=\chi+i \eta$ is a complex scalar field and

$$
\tilde{\mathcal{F}}_{\mu \nu}^{i}=\frac{1}{2} \sqrt{-g} \epsilon_{\mu \nu \rho \sigma} \mathcal{F}^{i \rho \sigma}
$$

We mention in passing that the action (1) is manifestly invariant under global $O(d, d+n)$ transformations:

$$
\begin{aligned}
& M \rightarrow \Omega M \Omega^{T}, \quad \Omega \in O(d, d+n) \\
& \Phi \rightarrow \Phi, g_{\mu \nu} \rightarrow g_{\mu \nu}, \quad B_{\mu \nu} \rightarrow B_{\mu \nu}, \quad \mathcal{A}_{\mu}^{i} \rightarrow \Omega_{j}^{i} \mathcal{A}_{\mu}^{j} .
\end{aligned}
$$

The equations of motion corresponding to $\Psi, g_{\mu \nu}$ and $A_{\mu}$ derived from the action (4) are

$$
\begin{gathered}
\frac{\nabla_{\mu} \nabla^{\mu} \Psi}{\eta^{2}}+i \frac{\nabla_{\mu} \Psi \nabla^{\mu} \Psi}{\eta^{3}}-\frac{i}{4} \mathcal{F} M \mathcal{F}+\frac{1}{4} \mathcal{F} \mathcal{L} \tilde{\mathcal{F}}+i \frac{2 \Lambda}{\eta^{2}}=0 \\
R_{\mu \nu}-\frac{\nabla_{\mu} \Psi \nabla_{\nu} \bar{\Psi}}{2 \eta^{2}}+\frac{1}{8} \operatorname{Tr}\left(\partial_{\mu} M^{-1} \partial_{\nu} M\right)-\frac{\eta}{2} \mathcal{F}_{\mu \lambda} M^{-1} \mathcal{F}_{\nu}{ }^{\lambda}+g_{\mu \nu}\left(\frac{\eta}{8} \mathcal{F} M^{-1} \mathcal{F}-\frac{\Lambda}{\eta}\right)=0 \\
\nabla_{\mu}\left(\eta(M \mathcal{L})_{i j} \mathcal{F}^{j \mu \nu}-\chi \tilde{\mathcal{F}}^{i \mu \nu}\right)=0
\end{gathered}
$$

and the Bianchi identity is

$$
\nabla_{\mu} \tilde{\mathcal{F}}^{i \mu \nu}=0
$$

The S-duality transformations correspond to

$$
\begin{aligned}
& \Psi \rightarrow \frac{a \Psi+b}{c \Psi+d}, \quad a d-b c=1, \quad a, b, \cdots \in \mathbf{Z}, \\
& \mathcal{F}_{\mu \nu}^{i} \rightarrow c \eta(M \mathcal{L})_{i j} \tilde{\mathcal{F}}_{\mu \nu}^{j}+(c \chi+d) \mathcal{F}_{\mu \nu}^{i}
\end{aligned}
$$

and the metric $g_{\mu \nu}$ and moduli $M$ remain invariant.

Explicit calculations show that under S-duality the terms in eqs.(7) and (8) remain invariant when $\Lambda=0$; however for nonvanishing $\Lambda$ these equations are not S-duality invariant. In this context, we mention that it has been observed [9], in specific cases, that S-duality invariance of equations of motion is broken in presence of $\Lambda$. To analize S-duality invariance of eqs. (7) and (8), let us consider a specific transformation ( $a=d=0, b=-c=1)$ 


$$
\Psi \rightarrow-1 / \Psi \text { and } \mathcal{F}_{\mu \nu}^{i} \rightarrow-\eta(M \mathcal{L})_{i j} \tilde{\mathcal{F}}_{\mu \nu}^{j}-\chi \mathcal{F}_{\mu \nu}^{i}
$$

Now it is straightforward to find that first four terms on the left-hand-side of (7) are invariant under above transformation (12) while the last term with $\Lambda$ is not. Similarly, it can also be checked that except $\Lambda$-term all other terms in eq.(8) make an invariant combination. Thus in general, the presence of cosmological constant breaks the S-duality invariance of the string equations of motion. Furthermore, in principle the cosmological constant $\Lambda$ can be generalised to a nontrivial dilaton potential $V(\Phi)$ which might be generated due to nonperturbative effects. However, the corresponding equations of motion are S-duality invariant only if $V(\Phi)=0$. We write the equations of motion involving $V(\Phi)$ ( after rescaling to Einstein metric ):

$$
\begin{array}{r}
\frac{\nabla \mu \nabla^{\mu} \Psi}{\eta^{2}}+i \frac{\nabla \mu \Psi \nabla^{\mu} \Psi}{\eta^{3}}-\frac{i}{4} \mathcal{F} M \mathcal{F}+\frac{1}{4} \mathcal{F} \mathcal{L} \tilde{\mathcal{F}}+i\left(\frac{2 \tilde{V}(\eta)}{\eta^{2}}-\frac{2}{\eta} \frac{\partial \tilde{V}(\eta)}{\partial \eta}\right)=0 \\
R_{\mu \nu}-\frac{\nabla_{\mu} \Psi \nabla_{\nu} \bar{\Psi}}{2 \eta^{2}}+\frac{1}{8} \operatorname{Tr}\left(\partial_{\mu} M^{-1} \partial_{\nu} M\right)-\frac{\eta}{2} \mathcal{F}_{\mu \lambda} M^{-1} \mathcal{F}_{\nu}{ }^{\lambda}+g_{\mu \nu}\left(\frac{\eta}{8} \mathcal{F} M^{-1} \mathcal{F}-\frac{\tilde{V}(\eta)}{\eta}\right)=0
\end{array}
$$

where $\tilde{V}(\eta)$ is the dilaton potential reexpressed in terms of new variable $\eta=e^{-\Phi}$. We note that the above equations of motion (13) are not invariant under the transformation (12) as long as the dilaton potential $V(\Phi)$ is nonzero.

Now let us consider an example [10] of six dimensional target space constructed by taking a tensor product of two WZW theories with the groups $S L(2, R)$ and $S U(2)$ respectively. Thus the underlying conformal field theory describing the above target space is exact. If we compactify one coordinate, say $\varphi$, of $S L(2, R)$ and another $\zeta$ of $S U(2)$ on torii then the resulting theory has a metric with $(-+++)$ Minkowski signature. A pair of gauge fields $\mathcal{A}_{\mu}^{(1) \alpha}(\alpha=\varphi, \zeta)$ appear from the metrics of the two groups and another pair of gauge fields $\mathcal{A}_{\mu, \alpha}^{(2)}$ come from the antisymmetric tensor fields. The scalar multiplet consists of a pair from the moduli and the dilaton. The exact conformal field theory backgrounds that satisfy equations of motion of four dimensional string effective action (4) are

$$
d s^{2}=-\left(\frac{r^{2}}{K_{S L}}-M+\frac{J^{2}}{4 r^{2}}\right) d t^{2}+\left(\frac{r^{2}}{K_{S L}}-M+\frac{J^{2}}{4 r^{2}}\right)^{-1} d r^{2}+\frac{K_{S U}}{4}\left(d \theta^{2}+\sin ^{2} \theta d \phi^{2}\right)
$$




$$
\begin{aligned}
& \mathcal{A}_{\mu}^{(1) \varphi}=\left(-\frac{J^{2}}{2 r^{2}}, \quad 0, \quad 0, \quad 0\right), \quad \mathcal{A}_{\mu}^{(1) \zeta}=\left(\begin{array}{llll}
0, & 0, & 0, & m \cos \theta
\end{array}\right),
\end{aligned}
$$

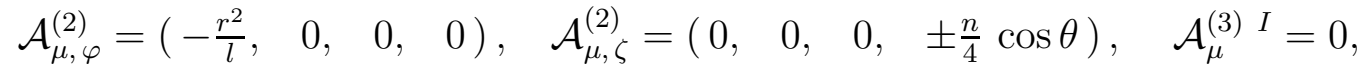

$$
\begin{aligned}
& G_{\alpha \beta}=\left(\begin{array}{cc}
r^{2} & 0 \\
0 & \frac{n}{4 m}
\end{array}\right), \quad B_{\alpha \beta}=0, \quad \Phi=-\ln r+\text { const. }, \quad H_{\mu \nu \sigma}=0,
\end{aligned}
$$

where $\alpha, \beta$ run over two compactified directions $\varphi$ and $\zeta$. Here $G_{\varphi \varphi}=r^{2}$ and $G_{\zeta \zeta}=n / 4 m$ correspond to the moduli. The corresponding six dimensional string effective action has graviton, antisymmetric tensor and dilaton only.

We mention in passing that the compactification of the direction $\zeta$ gives rise to a $U(1)$ gauge field with magnetic charge $m$ and modular invariance imposes the constraint $m n=$ $K_{S U}$. Notice that large $K_{S U}$ and $K_{S L}$ limits corresponds to Bertotti-Robinson [11 space time in four dimensions. This solution describes the throat limit of extremal dilaton black holes with electric and magnetic charge investigated by Kallosh et. al. [12 and also describes the throat limit of the Reissner-Nordstrom black hole. We recall that large $K_{S U}$ and $K_{S L}$ can be envisaged as the classical limit since these constants play the role of $1 / \hbar$ in the WZW theory. In this limit, the cosmological constant $\Lambda \rightarrow 0$.

Next, we present another way to obtain the four-dimensional black hole solutions. In this case, instead of compactifying the coordinate $\varphi$ of the $S L(2, R)$ alluded to above, we gauge the $U(1)$ subgroup. The gauged $S L(2, R)$ WZW action can be written in light cone coordinates $(z, \bar{z})$

$$
S(U, A)=S(U)+\frac{K_{S L}}{2 \pi} \int d^{2} z \operatorname{Tr}\left[U^{-1} \partial_{z} U A_{\bar{z}}+\partial_{\bar{z}} U U^{-1} A_{z}+U^{-1} A_{z} U A_{\bar{z}}+A_{z} A_{\bar{z}}\right]
$$

where $U(x) \in S L(2, R) \forall x$ in the manifold $M$. Integrating out the gauge fields in (15) by taking care of the Jacobian in the corresponding path-integral, one gets the two dimensional target space configuration [13]. As a result, the effective theory is a five-dimensional one with an appropriate cosmological constant term. The corresponding four dimensional effective 
action will arise from the compactification of this five-dimensional theory. In this prescription there are only two gauge fields, namely one comming from the metric and the other from the antisymmetric tensor field when $\zeta$ coordinate of $S U(2)$ is compactified. The background field configurations are:

$$
\begin{aligned}
& d s^{2}=-\left(1-\frac{M}{r}\right) d t^{2}+\left(1-\frac{M}{r}\right)^{-1} \frac{K_{S L} d r^{2}}{8 r^{2}}+\frac{K_{S U}}{4}\left(d \theta^{2}+\sin ^{2} \theta d \phi^{2}\right), \\
& \mathcal{A}_{\mu}^{(1) \zeta}=(0, \quad 0, \quad 0, \quad m \cos \theta), \quad \mathcal{A}_{\mu, \zeta}^{(2)}=\left(0, \quad 0, \quad 0, \quad \pm \frac{n}{4} \cos \theta\right), \quad \mathcal{A}_{\mu}^{(3) I}=0 \\
& G_{\zeta \zeta}=\frac{n}{4 m}, \quad \Phi=-\ln r+\text { const. }, \quad H_{\mu \nu \lambda}=0 .
\end{aligned}
$$

This background configuration (16) describes a four dimensional magnetically charged static black hole solution. In the asymptotic limit, i.e. $r \rightarrow \infty$, the topology of the spacetime is $R^{1} \times R^{1} \times S^{2}$.

These solutions (14) and (16) satisfy the background field equations of four dimensional string effective action with nonvanishing cosmological constant terms, $2 / K_{S U}-$ $2 / K_{S L}$ and $2 / K_{S U}-4 / K_{S L}$ respectively, which indeed break S-duality symmetry of the equations of motion. The scalar curvatures corresponding to these background configurations are respectively given by

$$
\frac{8}{K_{S U}}-\frac{2}{K_{S L}}-\frac{3 J^{2}}{2 r^{4}} \text { and } \quad \frac{8}{K_{S U}}+\frac{8 M}{K_{S L}} \frac{1}{r} .
$$

In summarizing, we have explored the consequences of S-duality transformations on the equations of motion with nonzero cosmological constant. First, we studied a four dimensional action in a general frame-work. The reduced action (1) could have been obtained from toroidal compactification of a heterotic string effective action in higher dimensions. Although these actions do not necessarily represent supersymmetric theories, S-duality invariance would have implied the absence of cosmological constant. We note that the cosmological constant term breaks S-duality for the exact conformal field theory backgrounds and is also responsible for nonvanishing of $R$ for asymptotically large $r$. 
In this context, let us briefly discuss the presence of higher order terms in $\alpha^{\prime}$ and the consequences of the S-duality transformations in the equations of motion. We write down the next higher order term in $\alpha^{\prime}$ [14 to the low energy string effective action (4) as

$$
S^{\prime}=\int d^{4} x \sqrt{-g} \eta\left(R_{\mu \nu \lambda \rho} R^{\mu \nu \lambda \rho}\right)
$$

In presence of the higher order term the equation of motion (7) gets an additional contribution

$$
\frac{i}{4} R_{\mu \nu \lambda \rho} R^{\mu \nu \lambda \rho}
$$

and similarly eq.(8) is modified with the extra term

$$
\eta\left[G_{\mu \nu}+g_{\mu \nu} \nabla_{\alpha} \nabla^{\alpha} R\right]
$$

where

$G_{\mu \nu}=-\frac{1}{2} g_{\mu \nu} R_{\alpha \beta \lambda \rho} R^{\alpha \beta \lambda \rho}+2 R_{\mu \alpha \beta \gamma} R_{\nu}^{\alpha \beta \gamma}-4 \nabla_{\alpha} \nabla^{\alpha} R_{\mu \nu}+2 \nabla_{\mu} \nabla_{\nu} R-4 R_{\mu \alpha} R_{\nu}^{\alpha}+4 R_{\mu \alpha \nu \beta} R^{\alpha \beta}$.

We have checked that under the S-duality transformation (12), eq.(7) with the additional term also breaks S-duality invariance. The graviton equation along with the higher order correction term as mentioned above is also not invariant under the S-duality. Thus it can be argued that the presence of the higher order terms do not restore the S-duality invariance in the equations of motion. Notice that when we dimensionally reduce the terms involving quadratic in curvature, there will be additional terms in (17) involving moduli and gauge fields ( arising from dimensional reduction ). We have seen that the contribution of (17) to equations of motion already breaks the S-duality. Therefore, even if we explicitly take into account the contribution coming from moduli and extra gauge fields in the corresponding equations of motion, the S-duality invariance will not be restored.

In this optics, we propose that vanishing of $\Lambda$ is closely related with the S-duality symmetry of string theory if we adopt the "naturalness" hypothesis. In this context, $\Lambda$ plays a dual role. From the macroscopic point of view, its smallness in cosmological observations is intimately related to the fact that our Universe is big and quite flat and in that sense it 
tells us about the physics at very large length scale. On the other hand, when we envisage it from a microscopic point of view, $\Lambda$ plays the role of a coupling constant in the string action and it is expected that quantum gravity considerations will provide us with an answer to its vanishing value. In the past, there have been several attempts [4, 15 to explain the smallness of $\Lambda$. Notable among them, the proposal of Coleman [16] in sequel to the works of Hawking [17] and Baum [18] has been the focus of attention. However, the mechanism proposed by Coleman to show how the cosmological constant vanishes has received some criticism [19]. If we construct theories which respect supersymmetry ( SUSY) and/or supergravity ( SUGRA ), then $\Lambda$ must be zero; however, these are not exact symmetries and thus are unable to explain why $\Lambda$ is so small [4]. We are aware that models with SUSY, require the SUSY breaking scale to be of order $\mathrm{TeV}$ and there will be contributions to $\Lambda$ from this breaking. Our proposal for resolution of the cosmological constant problem is based on two important hypothesis, i.e. S-duality is an exact symmetry of string theory and the "naturalness". We recall that the cosmological constant, although vanishingly small, does

not satisfy the "naturalness" criterion when treated in the framework of Einstein gravity. However, in the setting of string theory, we find it exceedingly attractive that $\Lambda$ does fulfill the criterion of naturalness when we incorporate S-duality as a symmetry. S-duality as an exact symmetry of string theory might have far reaching consequences than that meets the eye.

\section{ACKNOWLEDGMENTS}

Author, S.K. would like to thank Institute of Physics, where most of the work was done. One of us (J.M.) is grateful to John Schwarz for sharing his insights on S-duality and for valuable correspondence. Work of J.M. is supported by Jawaharlal Nehru Fellowship from Jawaharlal Nehru Memorial Fund. 


\section{REFERENCES}

[1] A. Font, L. Ibanez, D. Lust and F. Quevedo, Phys. Lett. B249, 35 (1990); S.J. Rey, Phys. Rev. D43, 526 (1991); S. Kalara and D. Nanopoulos, Phys. Lett B 267, 343 (1991). A. Shapere, S. Trivedi and F. Wilczek, Mod. Phys. Lett. A6, 2677 (1991); A. Sen, Nucl. Phys. B404, 109 (1993), Phys. Lett. B303, 22 (1993), Mod. Phys. Lett. A8, 2023 (1993); J. Schwarz and A. Sen, B312, 105 (1993), Nucl. Phys. B411, 35 (1994); P. Binetruy, Phys. Lett. B315, 80 (1993); A. Sen, Int. J. Mod. Phys. A9, 3707 (1994), Phys. Lett. B329, 217 (1994); J. Gauntlett and J. Harvey, preprint EFI-94-36; L. Girardello, A. Giveon, M. Porrati and A. Zaffaroni, preprint NYU-TH-94/06/02, NYU-TH-94/12/01; M. Duff and R. Khuri, Nucl. Phys. B411, 473 (1994); J. Schwarz, preprint CALT-68-1965; J. Harvey, G. Moore and A. Strominger, preprint EFI-95-01; A. Giveon, M. Porrati and E. Ravinovici Phys. Rep.244C, 77(1994); J. H. Schwarz, Proceedings of Workshop on Physics at the Planck Scale, Puri, December 1994; E. Witten, preprint IASSNS-HEP-95-18, hep-th/9503124.

[2] C. Vafa and E. Witten, preprint HUTP-94-A017; N. Seiberg and E. Witten, Nucl. Phys. B426, 19 (1994); Nucl. Phys. B431, 484 (1994); A. Ceresole, R. D'Auria and S. Ferrara, Phys. Lett. B339, 71 (1994); M. Bershadsky, A. Johansen, V. Sadov and C. Vafa, preprint HUTP-95-A004; Earlier works in duality includes: C. Montonen and D. Olive, Phys. Lett. B72, 117 (1977); P. Goddard, J. Nyuts and D. Olive, Nucl. Phys. B125, 1 (1977); H. Osborn, Phys. Lett. B83, 321 (1979); E. Witten and D.Olive, Phys. Lett. B78, 97 (1978).

[3] G. 't Hooft, Under the Spell of Gauge Principle (World Scintific, Singapore, p352, 1994).

[4] S. Weinberg, Rev. Mod. Phys. 61, 1 (1989), this article provides beautiful exposition of cosmological constant problem.

[5] G. Moore, Nucl. Phys. B293, 139 (1987); E. Alvarez and M.A.R. Osorio, Z. Phys. C44, 89 (1989); E. Alvarez, L. Alvarez-Gaume and Y. Lozano, CERN-Th-7486/94, 
hep-th/9410237.

[6] E. Witten, IAS preprint IASSNS-HEP-95-51, hep-th/9506101.

[7] K. Becker, M. Becker, and A. Strominger, NSF-ITP-95-07, hep-th/9502107.

[8] J. Maharana and J. Schwarz, Nucl. Phys. B390, 4 (1993); S. Hassan and A. Sen, Nucl. Phys. B375, 103 (1993).

[9] J. Maharana and H. Singh, Phys. Lett. B ( in press ), hep-th/9506213; for related work see, I. Pinkstone, DAMTP R96-62, hep-th/9505147.

[10] D. Lowe and A. Strominger, Phys. Rev. Lett. 73, 1468 (1994); H. Horowitz and D. Welch, Phys. Rev. Lett. 71, 328 (1993); N. Kaloper, Phys. Rev. D48, 2598 (1993); for earliar work see; M. Banados, C. Teitelboim and J. Zanelli, Phys. Rev. Lett. 69, 1849 (1992).

[11] B. Bertotti, Phys. Rev. 116, 1331 (1959); I. Robinson, Bull. Acad. Polon. Sci. 7, 351 (1959).

[12] R. Kallosh, A. Linde, P. Ortin, A. Peet and A. Van Proeyen, Phys. Rev. D46, 5278 (1992); R. Kallosh and A. Peet, Phys. Rev. D46, 5223 (1992).

[13] E. Witten, Phys. Rev. D44, 314 (1991).

[14] C. G. Callan, D. Friedan, E. Martinec and M. Perry, Nucl. Phys. B262, 593 (1985); A. Giveon, E. Rabinovici and A. Tseytlin, Nucl. Phys. B409, 339 (1993).

[15] For detailed references and discussions see, Euclidean Quantum Gravity, Eds. G. W. Gibbons and S. W. Hawking (World Scientific, 1993).

[16] S. Coleman, Nucl. Phys. B310, 643 (1988); B 307, 854 (1988); J. Preskill, Nucl. Phys. B323, 141 (1989); B. Grinstein, Nucl. Phys. B321, 439 (1989).

[17] S. W. Hawking, Phys. Lett. B134, 403 (1984). 
[18] E. Baum, Phys. Lett. B133, 885 (1983).

[19] For review see J. Maharana, "Wormholes and cosmological constant" in proceedings Summer School in High Energy Physics and Cosmology, Eds. J. C. Pati, S. RandjbarDaemi, E. Sezgin and Q. Shafi (World Scientific, Singapore, 1991). 